\title{
Dynamic Determination of Phase Diagrams by Active Machine Learning
}

\author{
Ichiro Takeuchi ${ }^{1}$
}

${ }^{1 .}$ Department of Materials Science and Engineering, University of Maryland, College Park, MD.

We are using composition spread libraries to rapidly determine the phase diagrams of functional materials systems. Combinatorial thin film libraries spanning large compositional ranges can result in a variety of data types including X-ray diffraction patterns, magnetic hysteresis loops, electrochemical voltammograms, and Raman spectra [1]. Because of large volumes of disparate types of data, it is difficult to study the details of hundreds of data entries one by one, but at the same time it is important to be able to glean subtle features associated with composition change. To deal with this issue, we have been employing machine learning techniques to quickly reduce the large amount of data and distill them to meaningful information [2-6].

In this work, we demonstrate autonomous control of high-throughput experiments driven by an active learning algorithm to rapidly map structural phases and their boundaries on composition spread libraries. $\mathrm{X}$-ray diffraction is carried out on composition spread chips mapping pseudo-binary phase diagrams of transition metal substituted $\mathrm{VO}_{2}$. A clustering algorithm is used to delineate structural phase distribution based on similarity of diffraction data. Graph-based endmember extraction and labeling (GRENDEL) is used as the unsupervised machine learning algorithm for clustering. Starting at room temperature, after each subsequent diffraction measurement, the obtained structure information is used to update knowledge of structural phase distributions, and the instrument is automatically directed to measure the next successive spot which is predicted to best improve the accuracy of the learned structural phase distributions. Once the accuracy reaches a certain threshold, the algorithm directs the experiment to move to the next temperature, and diffraction is performed on selected compositions on the spread again. Because active learning algorithm projects the clustering results to subsequent measurements at higher temperatures, the accuracy of the phase diagram gets higher and higher. This work shows that phase diagram mapping including controlling temperature can be successfully folded into an active learning loop. The results so far indicate that the number of measurements can be reduced by a factor as large as 10 in order to arrive at the correct phase digram. Fig. 1 shows an example set of diffraction data which were taken during the active learning controlled experimental run to determine the compositiontemperature phase diagram of $\mathrm{W}$ doped $\mathrm{VO}_{2}$.

In this presentation, we will also discuss our other attempts to control synthesis and characterization using active learning. The work is funded by ONR and NIST, and it was carried out in collaboration with A. Gilad Kusne, B. De Cost, J. Hattrick-Simpers, and Y. Liang.

References:

[1] I. Takeuchi et al, Review of Scientific Instruments 76 (2005), p. 062223.

[2] C. J. Long et al, Review of Scientific Instruments 78 (2007), p. 072217.

[3] R. Dell'Anna et al, QSAR and Combinatorial Science 27 (2008), p. 171.

[4] C. J. Long et al, Review of Scientific Instruments 80 (2009), p. 103902.

[5] A.G. Kusne et al, Scientific Reports 4 (2014), p. 6367. 
[6] A. G. Kusne et al, Nanotechnology 26 (2015), p. 444002.

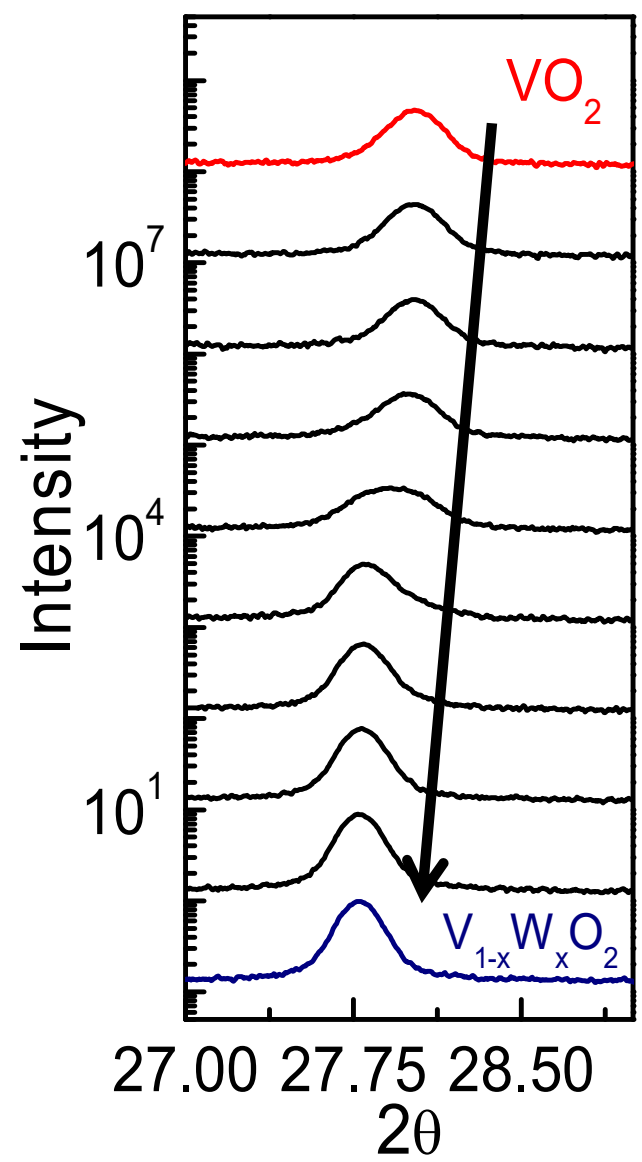

Figure. 1. Diffraction patterns taken at room temperature on $\mathrm{V}_{1-\mathrm{x}} \mathrm{W}_{\mathrm{x}} \mathrm{O}_{2}$ composition spread at room temperatures at different composition spots. Such diffractions patterns were used to demonstrate rapid phase diagram determination. Peak shifting and splitting are the key features used for phase grouping by clustering. 